\title{
Neotectónica de la falla Cipreses, Costa Rica
}

\author{
Evelyn Rodríguez ${ }^{1}$, Lepolt Linkimer ${ }^{1,2 *}$, Walter Montero ${ }^{3}$
}

DOI: http://dx.doi.org/10.18273/revbol.v41n2-2019001 @ ()

Forma de citar: Rodríguez, E., Linkimer, L., y Montero, W. (2019). Neotectónica de la falla Cipreses, Costa Rica. Boletín de Geología, 41(2), 15-33. DOI: 10.18273/revbol.v41n2-2019001.

\begin{abstract}
RESUMEN
La falla Cipreses se localiza en la parte central de Costa Rica, en la zona más poblada del país. En esta investigación se analiza esta falla a partir de aspectos geomorfológicos, geológicos y sismológicos. Las principales evidencias geomorfológicas encontradas son: escarpes, terrazas y ríos encajonados, desviados y represados. Geológicamente en los alrededores de la falla prevalecen tres litologías de edad pleistocena: tobas, brechas y lavas andesíticas, las cuales fueron encontradas desplazadas por la falla en un afloramiento. Del análisis sismológico se concluye que la sismicidad instrumental en la zona es escasa pero al menos un sismo ocurrido en el 2010 (Mw 4,1) fue originado en la falla Cipreses. La solución del mecanismo focal de este sismo es de tipo inverso, con un plano nodal de dirección de buzamiento N30 ${ }^{\circ} \mathrm{E} / 35^{\circ}$ consistente con el plano de falla observado en el campo y con las evidencias geomorfológicas. A partir de un modelo conceptual del fallamiento, se interpreta que la falla Cipreses corresponde con una falla maestra que se propaga hacia la superficie en dos segmentos de falla denominados Pinares y Guayabos. Los desplazamientos en estas fallas generan dos pliegues de tipo anticlinal que están expresados superficialmente como escarpes prominentes. Las evidencias recabadas permiten concluir que la falla Cipreses es activa, de tipo inverso, con una expresión en superficie de $14 \mathrm{~km}$ y con un potencial sísmico de magnitud Mw 5,9-6,5.
\end{abstract}

Palabras clave: Costa Rica; falla activa; falla Cipreses; neotectónica.

\section{Neotectonic of the Cipreses fault, Costa Rica}

\begin{abstract}
The Cipreses fault is located in the central part of Costa Rica, in the most populated region of the country. In this paper, the Cipreses fault is analyzed based on geomorphological, geological, and seismological observations. The main geomorphological evidences found are: scarps, terraces, river incisions, and deflected and dammed streams. In the vicinity of the fault, there are three main Pleistocene geologic units: tuff, breccia, and andesitic lavas. These units were found displaced by the fault in an outcrop. From the seismological analysis, we conclude that the instrumental seismicity is low, but at least one earthquake in 2010 (4.1 Mw) was originated in the Cipreses fault. The focal mechanism solution for this event indicates a thrust fault with a nodal plane of dip direction $\mathrm{N} 30^{\circ} \mathrm{E} / 35^{\circ}$ in agreement with the fault plane found in the field and the geomorphological observations. Based on a conceptual model for the faulting, we interpret that the Cipreses fault corresponds to a master fault that propagates to the surface along two fault segments named Pinares and Guayabos. The displacements of these faults made two anticlinal folds, which are expressed at the surface as prominent scarps. Based on the evidences found we conclude that the Cipreses fault is an active thrust fault with a surface expression of $14 \mathrm{~km}$ and with the seismic potential of magnitude Mw 5.9-6.5.
\end{abstract}

Keywords: Costa Rica; active fault; Cipresses Fault; neotectonics.

\footnotetext{
${ }^{1}$ Escuela Centroamericana de Geología, Universidad de Costa Rica, San José, Costa Rica. evelynroc15@gmail.com

${ }^{2}$ Red Sismológica Nacional, Universidad de Costa Rica, San José, Costa Rica. (*) lepolt.linkimer@ucr.ac.cr

${ }^{3}$ Centro de Investigaciones en Ciencias Geológicas, Universidad de Costa Rica, San José, Costa Rica. wmonteropo@gmail.com
} 


\section{INTRODUCCIÓN}

La zona central de Costa Rica se caracteriza por una alta densidad de fallas activas (Montero, 1994, 2001; Marshall et al., 2000). En esta zona, también se localiza la ciudad capital San José y la Gran Área Metropolitana (GAM). Costa Rica se localiza al sur del istmo centroamericano en una zona donde interactúan las placas del Coco, Caribe y Nazca y la microplaca de Panamá (FIGURA 1). La parte central de Costa Rica es atravesada por el Cinturón Deformado del Centro de Costa Rica (CDCCR), el cual ha sido interpretado como el límite entre la placa Caribe y la microplaca de Panamá (e.g. Marshall et al., 2000; Montero, 2001). El CDCCR incluye en una zona de $\sim 100 \mathrm{~km}$ de ancho, fallas y pliegues neotectónicos de diversa geometría que transfieren desplazamientos entre los bloques tectónicos de la zona (Montero, 1994, 2001; Marshall et al., 2000). El origen del CDCCR puede estar asociado con la colisión del levantamiento del Coco con el sureste de Costa Rica y la subducción de corteza oceánica engrosada, los cuales provocan el desplazamiento de la microplaca de Panamá con respecto de la placa Caribe (Marshall et al., 2000; Montero, 2001). Desde 1900, el CDCCR ha sido la fuente de 20 sismos destructivos incluyendo el que devastó la ciudad de Cartago en 1910 (6,1 Ms) que con un saldo de 300 víctimas, constituye el peor desastre de la historia de Costa Rica (Montero y Miyamura, 1981; Montero, 2010; Peraldo y Montero, 2010; Alonso-Henar et al., 2013; FIGURA 2).

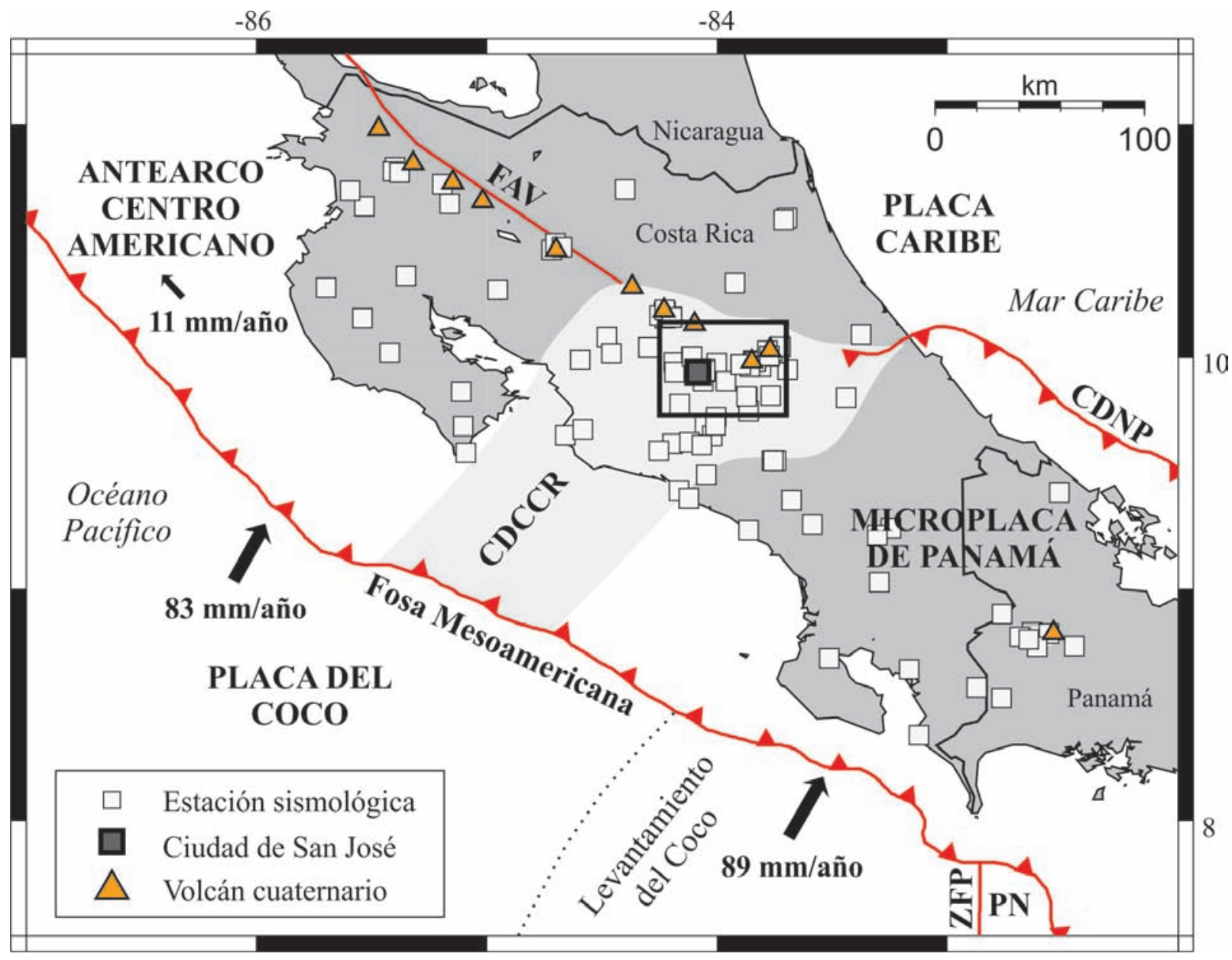

FIGURA 1. Marco tectónico de Costa Rica y ubicación de las estaciones de la RSN. La región de color gris claro representa el Cinturón Deformado del Centro de Costa Rica (CDCCR) de acuerdo con Montero y Rojas (2014). La línea roja rotulada con FAV representa las Fallas del Arco Volcánico de Guanacaste de acuerdo con Franco et al. (2012). Otros elementos tectónicos rotulados son: placa Nazca (PN) y Zona de Fractura de Panamá (ZFP). Los cuadrados blancos representan las estaciones sismológicas de RSN, OVSICORI-UNA, OSOP y UPA usadas en la relocalización de los sismos. Las flechas representan los vectores de velocidad de la placa del Coco de acuerdo con DeMets et al. (1994). El recuadro denota el área mostrada en la FIGURA 2. 


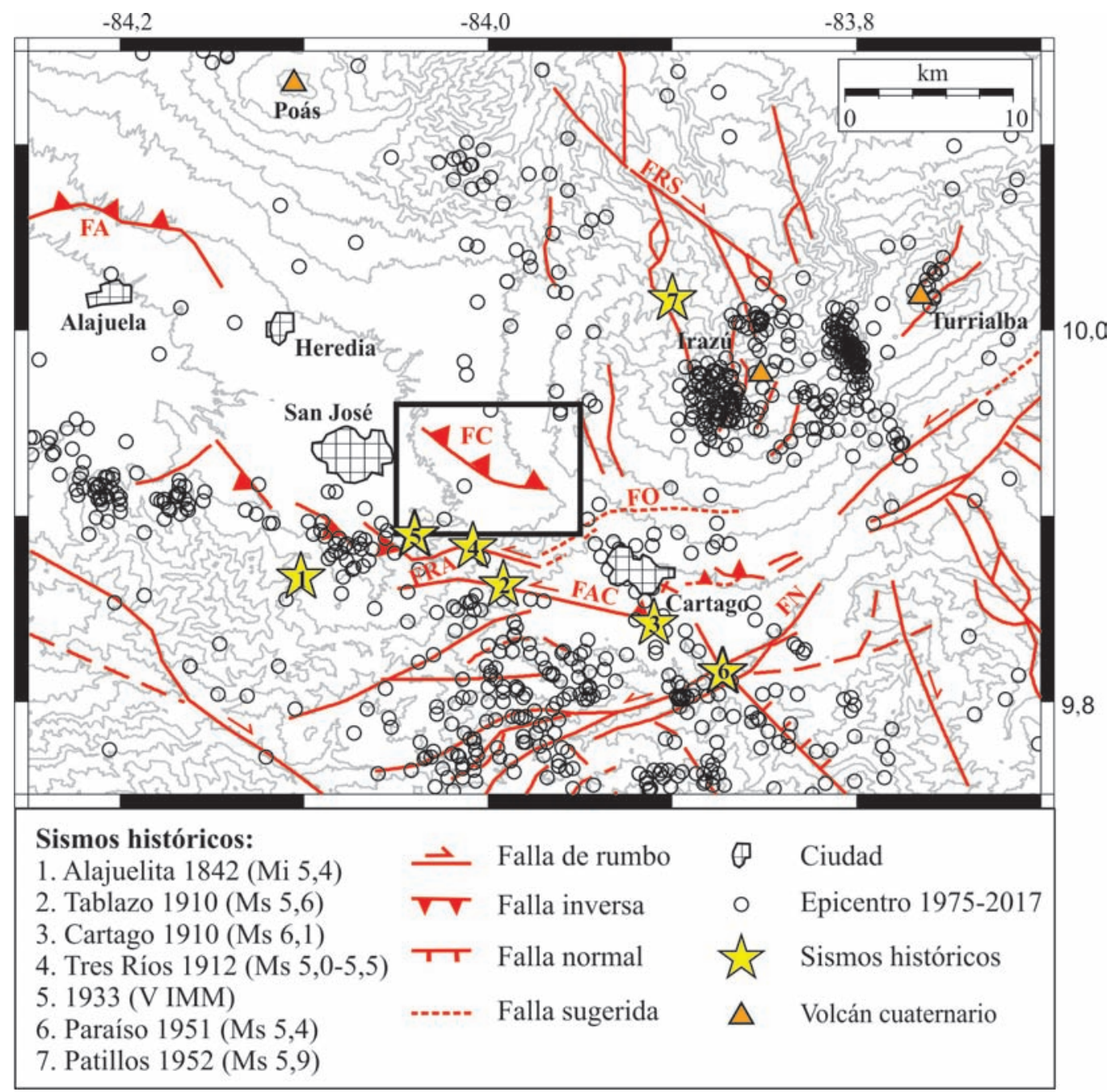

FIGURA 2. Sismicidad de mejor calidad localizada por la RSN entre los años 1975 y 2017. Las fallas activas son tomadas de Montero et al. (2013, 2016). Se rotularon las siguientes fallas: Alajuela (FA), Aguacaliente (FAC), Cipreses (FC), Navarro (FN), Ochomogo (FO), Río Azul (FRA) y Río Sucio (FRS). Las estrellas numeradas representan los terremotos históricos de acuerdo con Rojas (1993), Montero y Alvarado (1995), Ambraseys y Adams (2001), Fernández y Montero (2002) y Montero et al. (2005, 2016). El rectángulo denota el área mostrada en las FIGURAS 3, 4, 5 y 8.

La falla Cipreses, ubicada a sólo $5 \mathrm{~km}$ al este de San José, es un ejemplo de una de las fallas activas que conforman el CDCCR y además es una de las más próximas a la zona urbana (FIGURA 2). Esta falla ha sido descrita previamente en trabajos como los de Montero (2001), Fernández y Montero (2002), Denyer et al. (2003) y Montero et al. (2005), en los cuales los autores concuerdan en que esta estructura es una falla de tipo inverso. Otros estudios denominaron esta falla con el nombre de Granadilla como en WoodwardClyde Consultants (1993) y Fernández y Pacheco (1998). Alrededor de la falla Cipreses, existen otras fallas activas que también forman parte del CDCCR, como las fallas Alajuela, Aguacaliente, Navarro,
Ochomogo, Río Azul y Río Sucio (e.g. Borgia et al., 1990; Montero y Alvarado, 1995; Montero et al., 2005, 2016; FIGURA 2).

Geológicamente en la zona de la falla Cipreses se reconocen principalmente unidades de origen volcánico con edades que van desde el Pleistoceno hasta el Holoceno (Denyer y Arias, 1991; FIGURA 3) y en menor medida rocas sedimentarias del Mioceno Medio-Superior, como la Formación Coris (Fischer y Franco, 1979; FIGURA 3). La cobertura volcánica ha sido subdividida en varias formaciones llamadas: Reventado y Sapper y la Unidad Brechas (FIGURA 3). De acuerdo con Krushensky (1972), la Formación 
Reventado incluye tres miembros denominados Paraíso, Manto de Ceniza Roja y Superior, los cuales poseen las siguientes litologías: lava augítica (Miembro Paraíso), ceniza meteorizada (Miembro Manto de Ceniza Roja) y además coladas de lava andesíticas, depósitos de lahares y cenizas meteorizadas (Miembro Superior), con una edad de $136 \pm 5$ ka (Alvarado et al., 2006). La Formación Sapper incluye tefras, coladas de lava y diques (Krushensky, 1972) con edades de entre 68 y 36 ka (Allègre y Condomines, 1976). Finalmente, la Unidad Brechas, compuesta por los depósitos del Pleistoceno-Holoceno de los volcanes ubicados en la Cordillera Volcánica Central, incluye avalanchas de lodo y la cobertura de tobas y cenizas (Denyer y Arias, 1991), así como depósitos descritos como avalanchas de detritos (Alvarado et al., 2004; Hidalgo et al., 2004). Esta unidad se observa aflorando en casi todos los ríos y quebradas en los alrededores de la falla Cipreses, con litologías como brechas sobreyacidas por tobas (FIGURA 3).

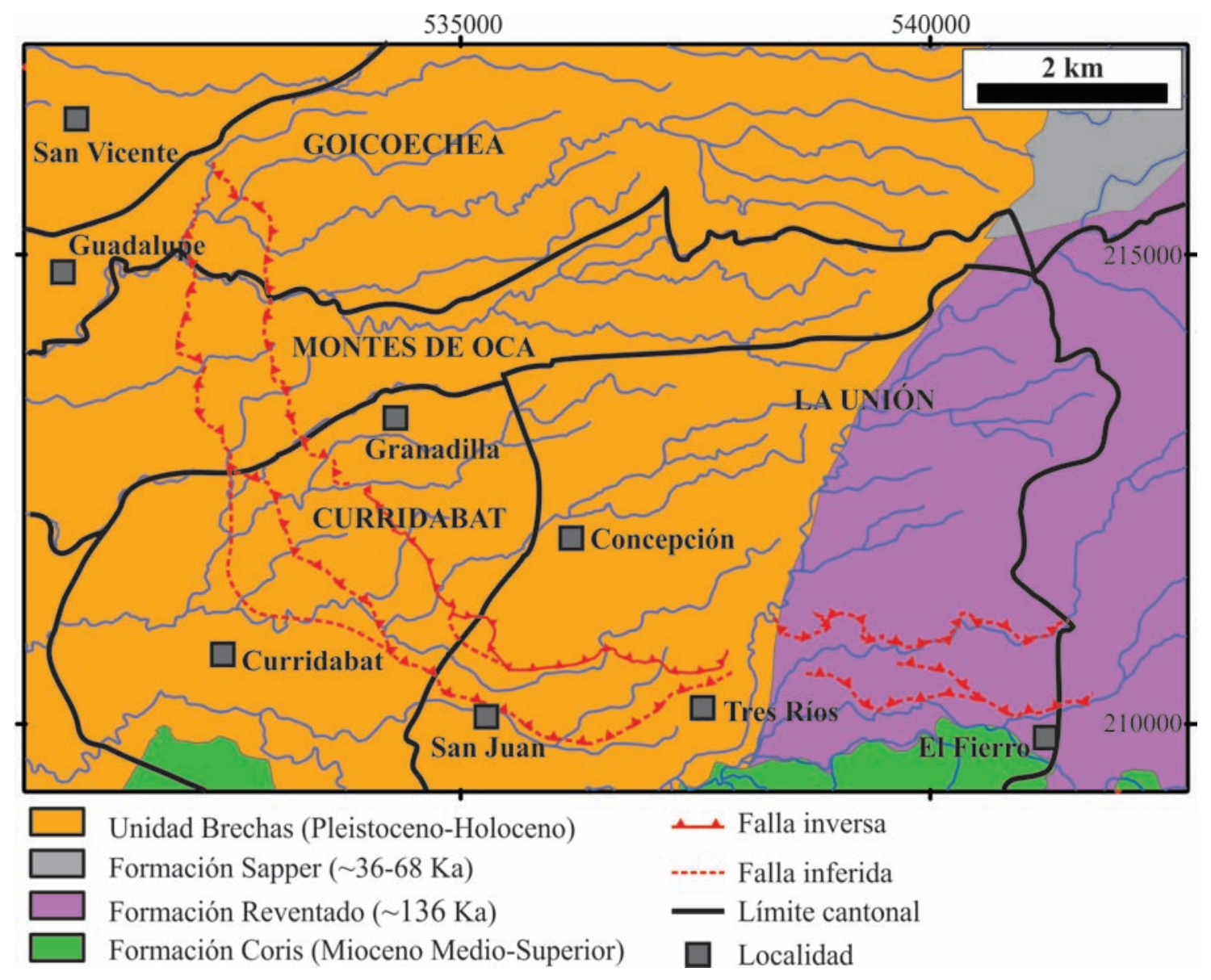

FIGURA 3. Mapa geológico de los alrededores de la falla Cipreses de acuerdo con Krushensky (1972), Fischer y Franco (1979) y Denyer y Arias (1991).

En este artículo se presentan los resultados del estudio de la falla Cipreses con base en aspectos geomorfológicos, geológicos y sismológicos. Además, se realiza una descripción de la geometría de la falla y se calcula su potencial sísmico. Aunque los sismos superficiales $(<30 \mathrm{~km})$ y de magnitud momento $(\mathrm{Mw})$ intermedia $(5,0-6,5)$ son frecuentes en la parte central de Costa Rica, la ciudad de San José hasta el año
2018 no ha sido el escenario de sismos destructivos con epicentros próximos a la zona urbana. Dado que la mayoría de la población y de las actividades socioeconómicas del país se concentran en la GAM, el estudio de las fallas activas en esta zona reviste de importancia para la apropiada determinación de la amenaza sísmica. 


\section{METODOLOGÍA}

La ubicación y características de la falla Cipreses se determinaron estudiando la geología, geomorfología y sismología de los alrededores de esta estructura. La zona de estudio comprende $96 \mathrm{~km}^{2}$ en los cantones de Montes de Oca, Curridabat, Goicoechea y La Unión ubicados en la parte central de Costa Rica (FIGURA 3).

Para el estudio geomorfológico se efectuó una búsqueda de imágenes aéreas y fotografías históricas con las que se hizo una fotointerpretación con estereoscopía con el fin de identificar elementos geomorfológicos asociados con el fallamiento activo. Se realizaron modelos de elevación digital con la base topográfica a escalas 1:10 000 y 1:50 000 del Instituto Geográfico Nacional de Costa Rica (IGN), con el que se llevó a cabo un análisis de pendientes. Además, se realizó un levantamiento de campo de los elementos geomorfológicos identificados en las fotos aéreas en busca de más evidencias de deformación neotectónica.

Para el análisis sismológico, se relocalizaron los sismos registrados por las estaciones de la Red Sismológica Nacional (RSN: UCR-ICE) cuyas localizaciones iniciales se encontraban en los alrededores de la falla Cipreses (FIGURA 2). La búsqueda en el catálogo de sismos de la RSN se efectuó entre los años de 1975 y 2017. Una vez revisados y relocalizados, se seleccionaron los de mejor calidad con base en criterios como un número mínimo de ocho estaciones, un gap de cobertura azimutal menor a $180^{\circ}$ y una estación cercana de entre 2 y $9 \mathrm{~km}$. La mayoría de los sismos de la base de datos inicial no cumplieron con los requisitos mínimos de calidad y luego de su relocalización, los hipocentros se ubicaron fuera de la zona estudiada para la falla Cipreses (rectángulo en FIGURA 2). La base de datos resultante fue de solo seis sismos, para los cuales, además de las estaciones de la RSN, fueron añadidos los sismogramas registrados por las estaciones del Observatorio Vulcanológico y Sismológico de Costa Rica (OVSICORI-UNA), el Observatorio Sismológico del Occidente de Panamá (OSOP) y la Universidad de Panamá (UPA). En total fueron usadas 111 estaciones de las mencionadas redes sismológicas (FIGURA 2).

Utilizando la última versión del Software SeisAn (Ottemöller et al., 2011), que incluye el programa HYP
(Lienert y Havskov, 1995), se realizó la relocalización de los eventos sísmicos a partir de la revisión cuidadosa de los arribos de las ondas $\mathrm{P}$ (primaria) y S (secundaria) y del uso de un modelo de velocidad de la onda $\mathrm{P}$ de siete capas y una razón de las velocidades de las ondas P y S (Vp/Vs) de 1,74. Además se realizó el cálculo de la magnitud momento (Mw) para cada sismo. Los mecanismos focales fueron elaborados con base en el primer arribo de la onda P, utilizando el programa FOCMEC (Snoke et al., 1984).

El trabajo de campo y la información geológica recopilada con base en bibliografía, se complementó con la información de pozos del Servicio Nacional de Aguas Subterráneas, Riego y Avenamiento (SENARA) de Costa Rica. Se recopiló la ubicación y las litologías de 30 pozos ubicados en la vecindad de la traza de la falla. Con esta información se realizó una interpretación que resultó en un modelo del fallamiento que integra todas las observaciones recabadas.

Finalmente, se efectuó una estimación sencilla del potencial sísmico de la falla usando algunas regresiones seleccionadas de la compilación realizada por Stirling et al. (2013) y consideradas apropiadas para el tipo de falla y contexto tectónico. Se utilizaron las relaciones propuestas por Wells y Coppersmith (1994), que relacionan el tamaño de un sismo ( $\mathrm{Mw}$ ) con la longitud (L) de la ruptura en superficie tanto para cualquier tipo de falla (Ecuación 1) como para fallas inversas (Ecuación 2), de acuerdo con las ecuaciones:

$$
\begin{gathered}
M w=5,08+1,16 \log (L) \\
M w=5,00+1,22 \log (L)
\end{gathered}
$$

Adicionalmente se utilizó la relación propuesta por Stirling et al. (2008) que relaciona la longitud (L) de la ruptura en superficie y el ancho de deformación (W) para fallas oblicuas, de acuerdo con la ecuación:

$$
M w=4,18+0,67 \log (W)+1,33 \log (L)
$$

Finalmente, se efectuó el mismo cálculo usando la relación propuesta por Wesnousky (2008) para fallas inversas, de acuerdo con la ecuación:

$$
M w=4,11+1,88 \log (L)
$$




\section{RESULTADOS}

\section{Geometría y expresión geomorfológica}

La falla Cipreses se compone de dos escarpes principales paralelos entre sí y separados por 700-900 m de distancia. En vista de planta estos escarpes tienen forma de " $L$ " y se extienden desde El Alto de Guadalupe hasta San Juan con rumbo noroeste-sureste y luego con rumbo este-oeste, hasta llegar a las proximidades de El Fierro (FIGURA 4A). Estos dos escarpes principales se describen en este apartado con el nombre de Pinares y Guayabos y lucen como los escarpes de flexura asociados con fallas inversas identificados por Audemard (2003) en los Andes de Venezuela. Geológicamente, en los escarpes Pinares y Guayabos se encuentran afloramientos de la Unidad Brechas descrita en la Introducción (FIGURA 3).

El escarpe Pinares es el más prominente, mira al suroeste o al sur, tiene una longitud de 14,1 km y una altura de entre 40 y $100 \mathrm{~m}$ (FIGURA 4B). Este escarpe presenta pendientes de entre $5^{\circ}$ y $14^{\circ}$ y se extiende desde $\mathrm{El}$ Alto de Guadalupe hasta El Fierro, pero su segmento de mayor altura se localiza entre San Juan y El Fierro (FIGURA 4A). Un elemento geomorfológico asociado al escarpe Pinares, está ubicado al sur de la localidad de Concepción. En este sector existe un contraescarpe de 15 $\mathrm{m}$ de altura y 2,6 km de longitud, que mira al noreste con una pendiente de entre $1^{\circ}$ y $3^{\circ}$ (FIGURA 4C).

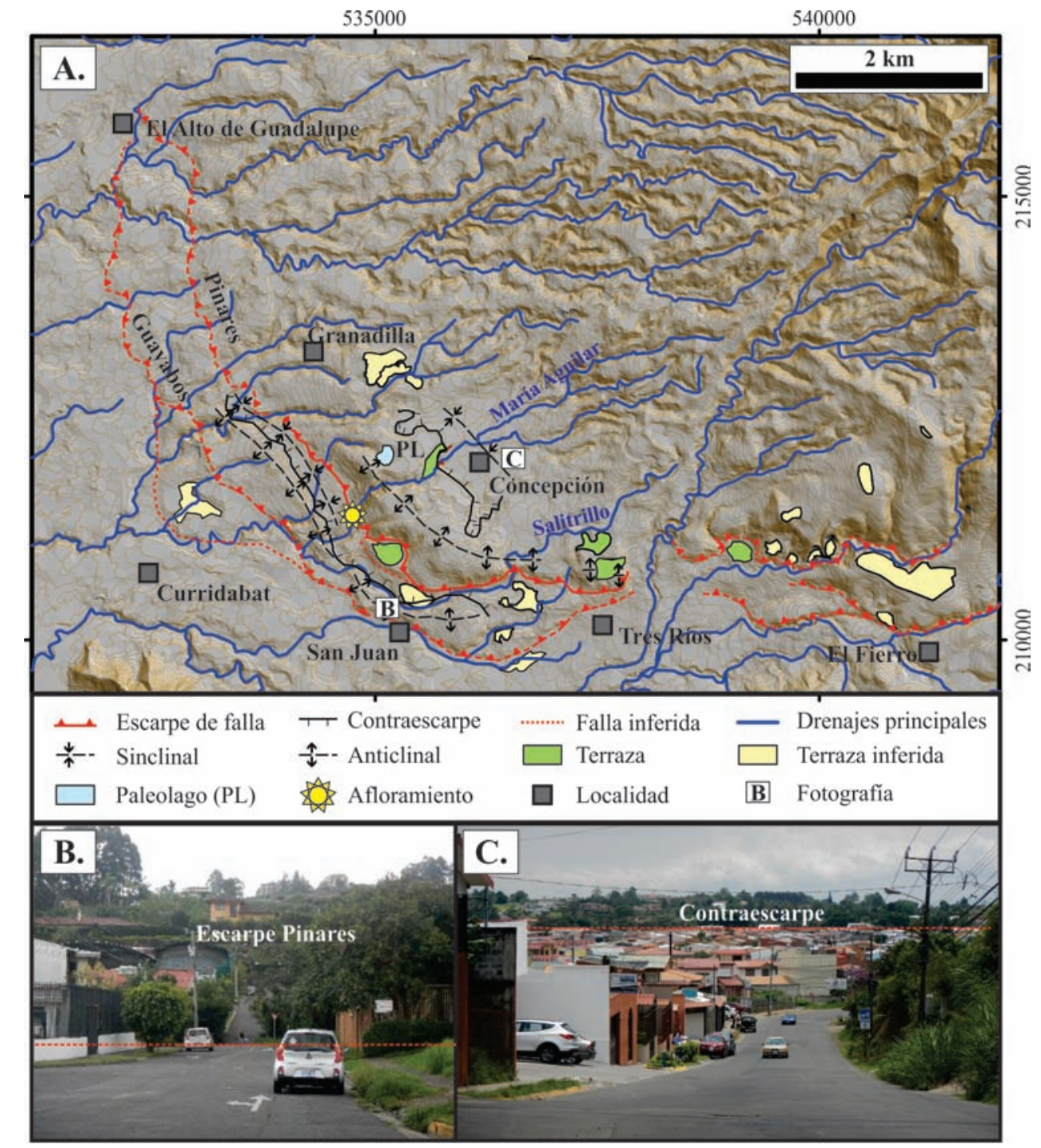

FIGURA 4. A. Elementos morfotectónicos principales de la falla Cipreses. Los cuadrados blancos rotulados con letras muestran los sitios en donde se tomaron las fotografías mostradas en las partes B y C de la figura. B. Escarpe Pinares visto hacia el noreste. La línea punteada roja muestra la base del escarpe. C. Contraescarpe visto hacia el suroeste a través de la localidad de Concepción. La línea punteada roja muestra la base del contraescarpe. 
El escarpe Guayabos mira también al suroeste o al sur, tiene una longitud de 14,1 km y una altura de entre 10 y $15 \mathrm{~m}$. Este escarpe presenta pendientes de entre $3^{\circ}$ a $30^{\circ}$ y se extiende desde El Alto de Guadalupe hasta El Fierro y al igual que el escarpe Pinares, posee poca expresión en el sector noroeste en las cercanías de El Alto de Guadalupe. Un elemento geomorfológico relacionado con el escape Guayabos, se ubica al noreste de Curridabat (en el sector de Guayabos) en donde existe un contraescarpe de 10 a $20 \mathrm{~m}$ de altura, que mira al noreste y que se extiende por $3,1 \mathrm{~km}$ con un rumbo noroeste-sureste y con una pendiente de unos $7^{\circ}$ (FIGURA 4A).

En los alrededores de los escarpes Pinares y Guayabo existen además 32 superficies de aplanamiento o terrazas fluviales. Estas terrazas fueron identificadas a partir de la observación geomorfológica, fotos aéreas y modelos de elevación digital, y varias de ellas fueron corroboradas durante el trabajo de campo (FIGURA 4A). El área de estas terrazas varía entre $14.000 \mathrm{~m}^{2} \mathrm{y}$ $282.000 \mathrm{~m}^{2}$, para un promedio por terraza de $46.000 \mathrm{~m}^{2}$. La mayoría de las terrazas se distribuyen al este y al norte del escarpe Pinares. En las zonas densamente pobladas, no fue posible comprobar el tipo de depósito que las constituyen por lo que se les clasificó como inferidas (FIGURA 4A).

En general, el sistema de drenaje presenta una dirección preferencial hacia el oeste en el sector norte del área de estudio y hacia suroeste en el sector sur (FIGURA 5). Existen anomalías en el patrón de drenaje como el encajonamiento en algunos de los ríos de mayor caudal, por ejemplo los llamados María Aguilar y Salitrillo, los cuales cortan los escarpes Pinares y Guayabos (FIGURA 5).

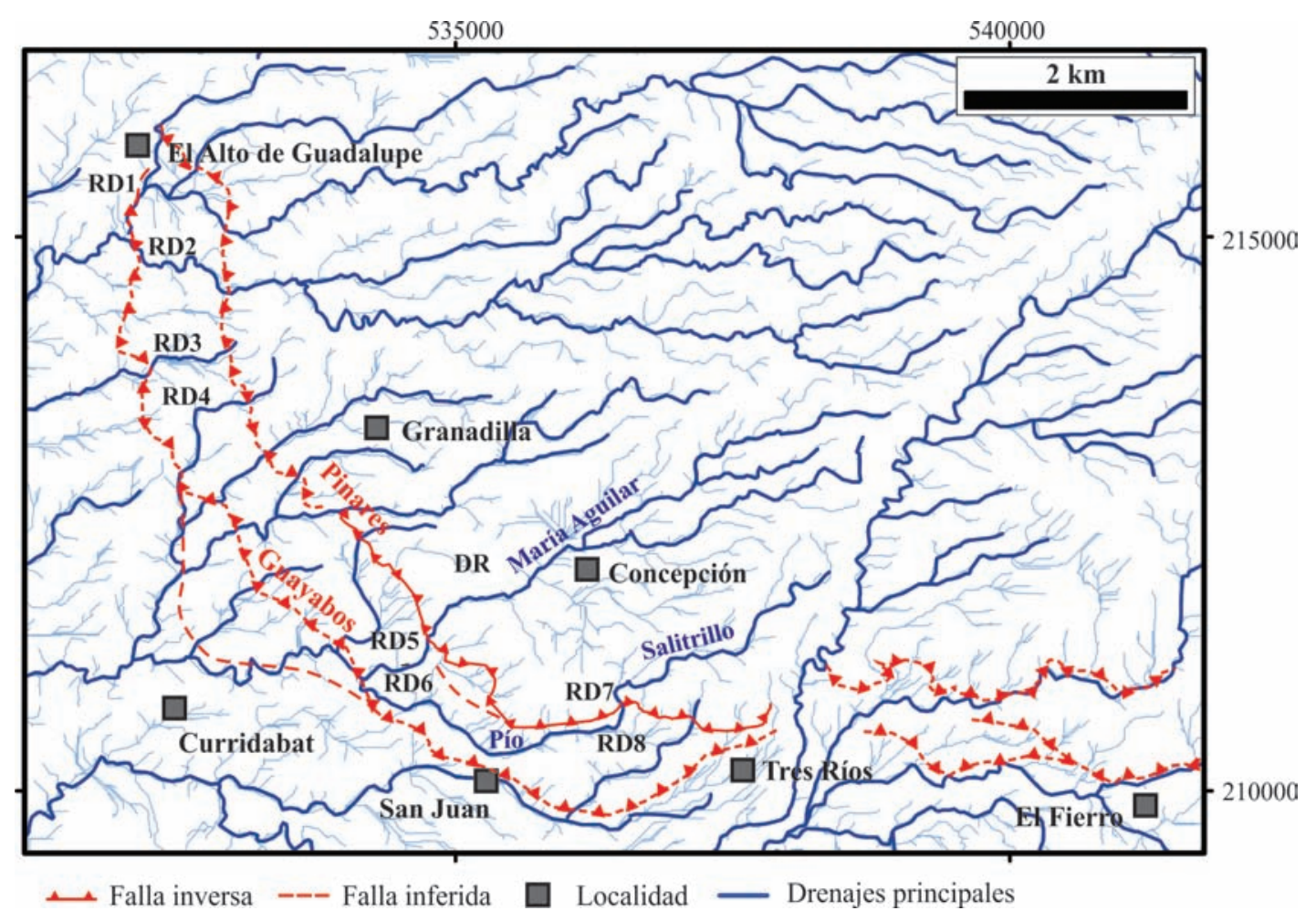

FIGURA 5. Mapa de drenajes. Los ríos principales se muestran como líneas más gruesas. Los ríos desviados (RD) mencionados en el texto son rotulados como RD1 a RD7 o con sus respectivos nombres. DR significa drenaje represado.

Otra anomalía es la presencia de un drenaje represado (DR, FIGURA 5) que pudo haber formado un paleolago (PL en FIGURA 4A) en las cercanías de Concepción. En este punto se observó en fotografías aéreas una depresión de unos 100 m de diámetro, la cual no pudo ser corroborada en el campo debido a la reciente construcción de una urbanización en esa zona. No obstante la información recabada de un pozo del SENARA (pozo AB-1681) ubicado en la zona de la depresión muestra 17 metros de arcillas y materia orgánica en la parte más superficial, por lo que se infiere que estos depósitos podrían corresponder con los de un paleolago. 
Además, existen drenajes que son desviados (RD), por ejemplo los rotulados como RD1, RD2, RD3 y RD4 (FIGURA 5) presentan desvíos en las proximidades de los escarpes Guayabos y Pinares. En particular, en algunos drenajes se presentan goteras tectónicas, es decir, un patrón de drenaje en el cual el río discurre paralelo al escarpe, similar al identificado y asociado con fallas inversas en Colombia y Venezuela (e.g. Audemard, 1999, 2003; Ollarves et al., 2006). Por ejemplo, el río señalado con RD5 (FIGURA 5), que inicia al oeste de Concepción discurre hacia el oeste, pero conforme transcurre a través del escarpe Pinares cambia a un rumbo sureste discurriendo paralelo a dicho escarpe. Aguas abajo, RD5 se curva hacia el este cuando se encuentra con el escarpe Guayabos en donde además el valle se profundiza.

El río María Aguilar (RD6 en FIGURA 5) atraviesa con dirección suroeste la localidad de Concepción. En este sector el río no ha profundizado su cauce, pero conforme avanza hacia el suroeste, el cauce empieza a profundizarse en las partes altas del escarpe Pinares. El río presenta además ligeros cambios de rumbo hasta llegar al escarpe Guayabos en donde cambia hacia el oeste, en forma similar al drenaje señalado como RD5.

Al atravesar el escarpe Guayabos, los drenajes RD5 y RD6 presentan un patrón de drenaje denominado en forma de rastrillo (broom-shaped river pattern). En este sector, los drenajes menores se encuentran ortogonalmente en un drenaje mayor que fluye aguas abajo atravesando el escape Guayabos (FIGURA 5). Un patrón similar ocurre con los drenajes RD1 y RD2 cuando transcurren por el mismo escarpe. Este tipo de patrón de drenaje ha sido identificado y asociado con fallas inversas en los Andes de Venezuela y Colombia (e.g. Audemard, 1999; Ollarves et al., 2006) y Argentina (Perucca et al., 2013; Zárate et al., 2014; Audemard et al., 2016).

El río Salitrillo inicia con un rumbo sureste al sur de Concepción (FIGURA 5), para luego cambiar de rumbo hacia el sur (RD7) cuando se encuentra con el escarpe Pinares y después retomar la dirección inicial hacia el suroeste, en la parte baja del escarpe Pinares. Luego del desvío, de acuerdo con la cartografía, el drenaje adquiere el nombre de río Pío (RD8). El drenaje continúa aguas abajo con un rumbo noroeste, entre los escarpes Pinares y Guayabo, para luego cambiar de rumbo hacia el suroeste cuando atraviesa el escarpe Guayabos.

\section{Expresión geológica}

Pese a que los afloramientos rocosos dentro del área de estudio son escasos, fue posible encontrar un sitio en el que existe una falla expuesta afectando depósitos recientes. Este sitio está ubicado en la margen izquierda del río María Aguilar, en las cercanías de Pinares de Curridabat (coordenadas Lambert 534766 E y 211375 N; FIGURAS 4A, 6 y 7). La falla se observó en un afloramiento de $10 \mathrm{~m}$ de largo y $5 \mathrm{~m}$ de alto, no obstante la cobertura vegetal en la parte superior y los depósitos aluviales en la parte inferior limitaron el seguimiento de la falla en esos sectores.

Las litologías encontradas en el sitio son tres principalmente: brechas, tobas y depósitos aluviales (FIGURA 6). Las brechas y las tobas pertenecen a la Unidad Brechas descrita en la Introducción (FIGURA 3). Las brechas se caracterizan por fragmentos centimétricos subangulares, principalmente de composición volcánica, inmersos en una matriz arcillolimosa color café o rojiza. Las tobas son de color café y se encuentran en el sector suroeste del afloramiento. Finalmente, los depósitos aluviales están constituidos por bloques redondeados centimétricos de composición volcánica, con muy poca matriz areno-limosa. Se infiere que las litologías del afloramiento son del PleistocenoCuaternario.

La falla observada en este afloramiento sobrecorre las brechas sobre los depósitos aluviales y las tobas (FIGURAS 6 y 7). Los depósitos aluviales se observan acuñados dentro de las tobas (FIGURA 6B). En el sector suroeste del afloramiento, las brechas sobreyacen a las tobas con un claro contacto de falla (FIGURA 7A). Otro detalle del afloramiento es que en la zona de contacto entre la brecha y el aluvión, se encontró una brecha ("harina") de falla color beige, con hasta $10 \mathrm{~cm}$ de espesor y caracterizada por pequeños fragmentos de roca triturada (FIGURA 7B).

El plano de falla medido en el afloramiento tiene una dirección de buzamiento de $\mathrm{N} 55^{\circ} \mathrm{E}$ y un ángulo de buzamiento que aumenta conforme se aproxima a la superficie y que varía entre $10^{\circ}$ y $25^{\circ}$ (FIGURA 6B).

También se reconocieron estrías de falla observadas en el bloque del techo en el sector donde la brecha está en contacto con la toba (FIGURA 7C). Allí se midió un ángulo de rake de $85^{\circ}$, el cual es concordante con el de una falla con muy alta componente de movimiento inverso. La falla encontrada en este afloramiento es denominada en este estudio como falla Pinares. 


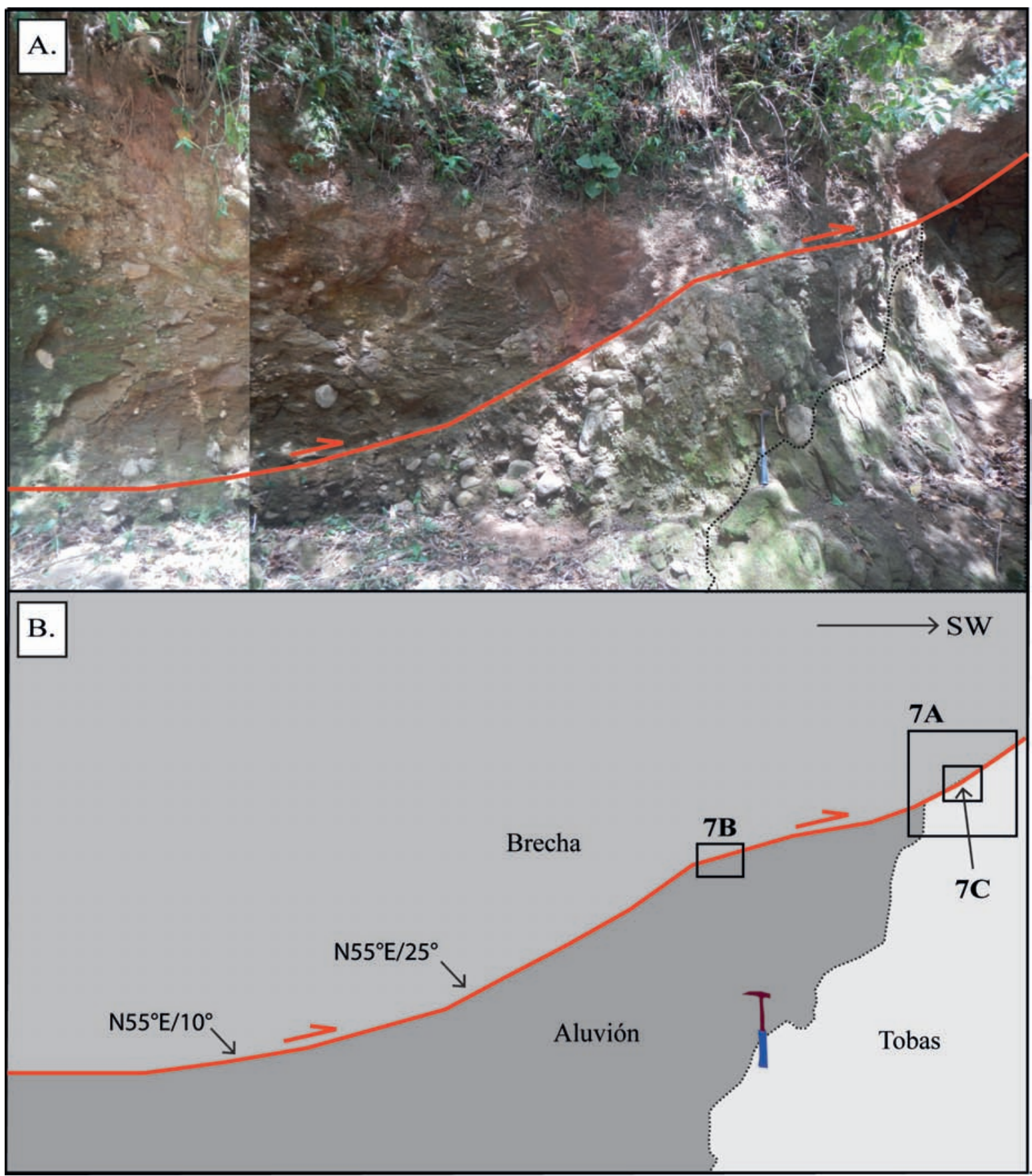

FIGURA 6. A. Afloramiento de la falla Pinares cuya ubicación se muestra en la FIGURA 4A. B. Esquema del afloramiento. Los rectángulos muestran las zonas detalladas en la FIGURA 7.

\section{Sismicidad}

Los sismos históricos más importantes con epicentro en la vecindad de la falla Cipreses (i.e., Rojas, 1993; Montero y Alvarado, 1995; Ambraseys y Adams, 2001; Fernández y Montero 2002; Montero et al., 2005, 2016; FIGURA 2) son los terremotos de Alajuelita del 21 de marzo de 1842 (con una magnitud Mi estimada con base en observaciones de intensidad de 5,4), Tablazo del 13 de abril de 1910 (Ms 5,6), Cartago del 4 de mayo de 1910 (Ms 6,1), Tres Ríos del 21 de febrero de 1912 (Ms 5,0-5,5), sureste de San José del 12 de julio de 1933 (Intensidad Mercalli Modificada IMM máxima de V), Paraíso del 22 de agosto de 1951 (Ms 5,4) y Patillos del 30 de diciembre de 1952 (Ms 5,9). 


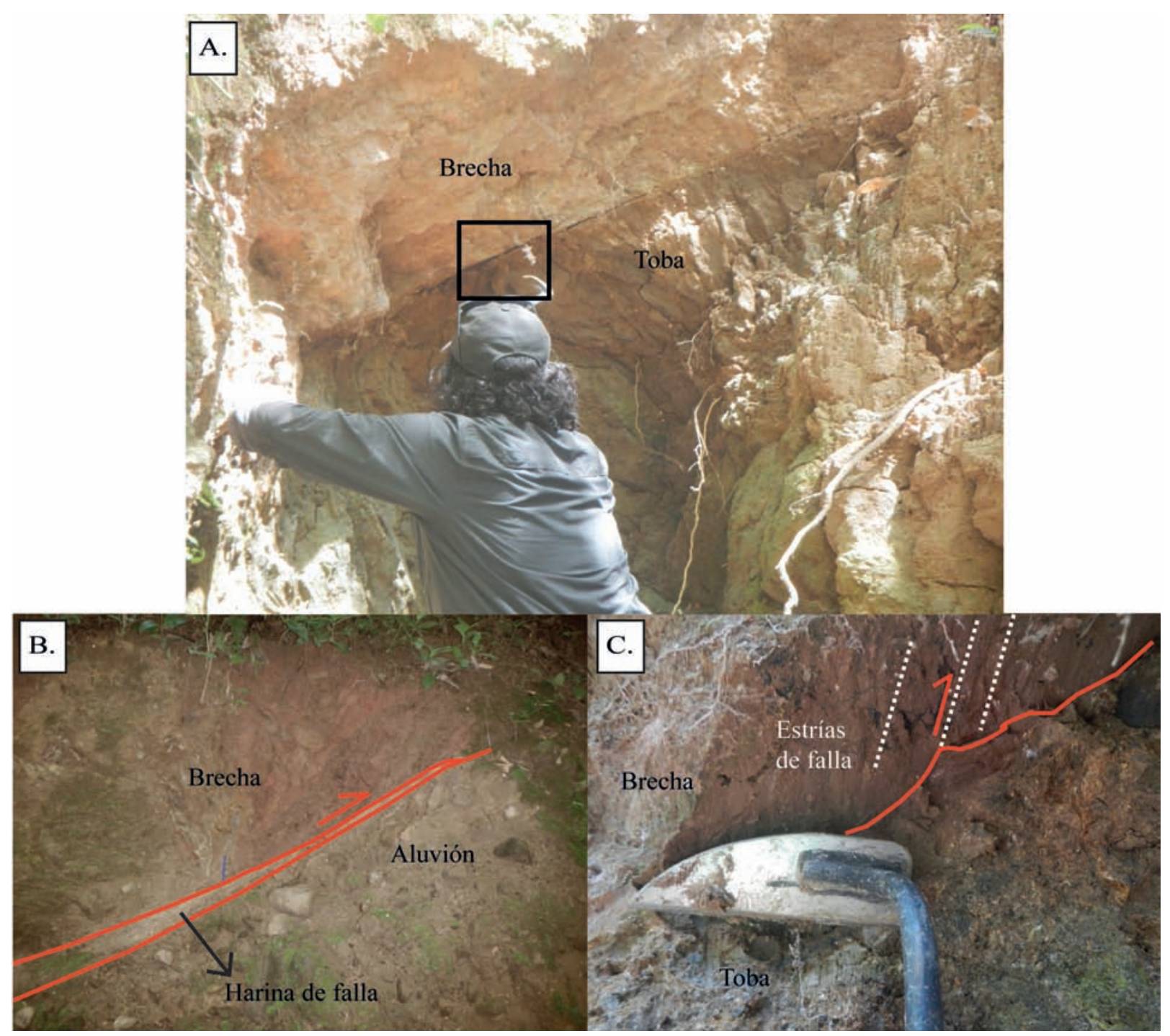

FIGURA 7. Detalle del afloramiento de la falla Pinares cuya ubicación se muestra en las FIGURAS 4A y 6. A. Contacto por falla entre brechas y tobas. Recuadro marca el detalle mostrado en la parte C. B. Zona con gouge ("harina”) de falla en el contacto entre la brecha y el aluvión. C. Zona con estrías de falla en el contacto entre la brecha y la toba. Las líneas punteadas muestran la dirección de las estrías.

De acuerdo con Montero et al. (2005), los eventos de 1912 y 1933 pudieron haberse originado en la falla Río Azul pues en esa zona se alcanzaron las intensidades más altas. No obstante, su origen sigue siendo incierto ya que se carece de una localización precisa de los hipocentros. Queda entonces abierta la posibilidad de que estos dos sismos se pudieron haber generado en la falla Cipreses, que se encuentra ubicada a solo $6 \mathrm{~km}$ al norte de la falla Río Azul (FIGURA 2).

La sismicidad instrumental en la zona de la falla Cipreses, contenida en el catálogo de la Red Sismológica Nacional (RSN: UCR-ICE) es baja en frecuencia y magnitud y dispersa (FIGURA 2). Montero et al. (2005) sugieren que algunos de los eventos ubicados en la zona este de San José pudieron haberse generado en la falla Cipreses. La FIGURA 2 muestra la sismicidad registrada por la RSN entre 1975 y 2017. Con el fin de mostrar los sismos localizados con la mejor calidad, en esta figura se muestran los que cumplen los siguientes criterios: un mínimo de ocho estaciones, una raíz media cuadrática (RMS) menor a 0,7 y una cobertura azimutal (GAP) de 180 grados. Se puede observar que la sismicidad es escasa en la vecindad de la falla Cipreses en contraste con otras zonas como los edificios volcánicos del Irazú y 
Turrialba y en las fallas ubicadas al sur de San José y Cartago, donde la sismicidad es abundante durante el periodo estudiado (FIGURA 2).

Para la relocalización de la sismicidad instrumental en la vecindad de la falla Cipreses fueron seleccionados todos los sismos registrados por la RSN en la zona y luego se siguieron los criterios detallados en la metodología. Solamente seis de los sismos relocalizados tienen su epicentro en la zona hacia donde buza el plano de falla ubicada al noreste de los escarpes Pinares y Guayabos (TABLA 1 y FIGURA 8). Estos sismos ocurrieron entre el 2010 y el 2015 y se caracterizan por una (Mw) de entre 2,6 a 4,1 y profundidades de entre $3 \mathrm{~km}$ y $8,9 \mathrm{~km}$.

TABLA 1. Características de la sismicidad relocalizada (ver FIGURA 8).

\begin{tabular}{cccccc}
\hline Número & Día/Mes/Año & Latitud & Longitud & Profundidad $(\mathbf{k m})$ & Mw \\
\hline 1 & $05 / 03 / 2010$ & 9,950 & $-84,016$ & 3,0 & 4,1 \\
2 & $13 / 03 / 2010$ & 9,938 & $-84,018$ & 6,1 & 3,1 \\
3 & $11 / 02 / 2013$ & 9,953 & $-83,975$ & 8,8 & 3,2 \\
4 & $12 / 02 / 2013$ & 9,964 & $-83,963$ & 8,9 & 3,0 \\
5 & $31 / 05 / 2015$ & 9,966 & $-84,003$ & 5,6 & 2,6 \\
6 & $20 / 12 / 2015$ & 9,957 & $-83,977$ & 6,2 & 2,8 \\
\hline
\end{tabular}

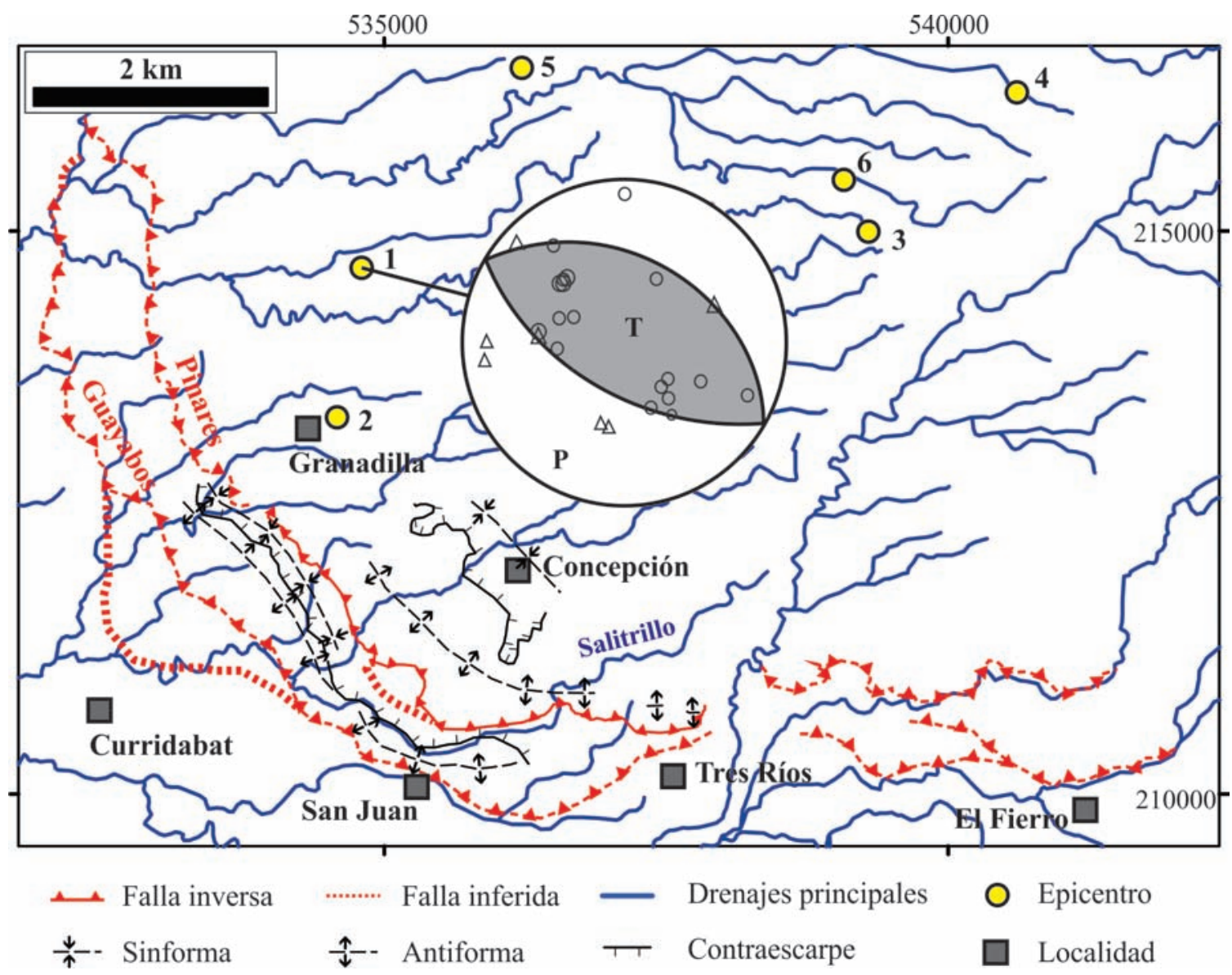

FIGURA 8. Falla Cipreses y sismos relocalizados numerados de acuerdo con la TABLA 1. Se muestra la solución del mecanismo focal del sismo del 5 marzo del 2010 (Mw 4,1) a 3 km de profundidad. En el mecanismo focal, la polaridad de los primeros arribos es representada por círculos para los casos de arribos positivos (compresión) y triángulos para los arribos negativos (dilatación). P y T representan los ejes de presión y tensión, respectivamente. 
El sismo de mayor magnitud (Mw 4,1) sucedió el 5 marzo del 2010 (número 1 en TABLA1). Este evento fue percibido con una IMM de $\mathrm{V}$ en la zona del epicentro y de forma leve en ciudades más distantes como Alajuela, Heredia y Cartago (RSN, 2010; FIGURA 2). El 13 de marzo del 2010 ocurrió una réplica de Mw 3,1 (número 2 en TABLA 1) que también fue percibida en la zona epicentral (RSN, 2010). Estos dos sismos del 2010 son los más cercanos a los escarpes Pinares y Guayabos (FIGURA 8).

De los seis sismos relocalizados solamente se logró calcular un mecanismo focal de buena calidad para el evento del 5 de marzo del 2010. La localización y cálculo del mecanismo focal se realizó a partir de la lectura de 47 arribos de la onda $\mathrm{P}, 27$ polaridades claras y un GAP de $58^{\circ}$. La estabilidad de la solución fue explorada para profundidades de entre 3 y $6 \mathrm{~km}$ (TABLA 2) en las cuales se tenían los menores residuos en la localización. En todos los casos, las soluciones corresponden con una falla inversa cuyos planos nodales tienen un rumbo noroeste-sureste, un ángulo de inclinación de entre 34 y $56^{\circ}$ y un ángulo de rake de entre 76 y $90^{\circ}$ (TABLA 2). Para todas las profundidades, uno de los planos nodales se inclina hacia el noreste (plano nodal 1, TABLA 2). Este plano nodal es consistente con la falla Cipreses.

TABLA 2. Solución del mecanismo focal del sismo del 5 de marzo del 2010 (Mw 4,1) para diferentes profundidades.

\begin{tabular}{ccccccccccc}
\hline $\begin{array}{c}\text { Profundidad } \\
(\mathrm{km})\end{array}$ & \multicolumn{3}{c}{ Plano nodal 1 } & \multicolumn{3}{c}{ Plano nodal 2} & \multicolumn{3}{c}{ Eje Presión } & \multicolumn{2}{c}{ Eje Tensión } \\
\hline 3 & Rum. & Buz. & Rake & Rum. & Buz. & Rake & Rum. & Buz. & Rum. & Buz. \\
4 & 120 & 35 & 90 & 120 & 55 & 90 & 210 & 10 & 30 & 80 \\
5 & 125 & 46 & 76 & 145 & 46 & 104 & 224 & 0 & 316 & 80 \\
6 & 124 & 34 & 90 & 124 & 56 & 90 & 214 & 11 & 34 & 79 \\
\hline
\end{tabular}

Buz. Buzamiento. Rum. Rumbo.

La solución a $3 \mathrm{~km}$ de profundidad fue considerada como la mejor por mostrar el menor RMS (0,36 s) (FIGURA 8). Los errores en la localización del sismo fueron de 1,2, 1,6 y 1,8 km en latitud, longitud y profundidad, respectivamente. Tomando en cuenta estas incertidumbres en la localización del hipocentro y el plano nodal que es consistente con la dirección de buzamiento de la falla Cipreses $\left(\mathrm{N} 30^{\circ} \mathrm{E} / 35^{\circ}\right.$ y ángulo de rake de $90^{\circ}$, TABLA 2), el hipocentro del sismo del 5 de marzo del 2010 podría coincidir con la ubicación de la falla en profundidad. Un cálculo trigonométrico sencillo usando la profundidad del hipocentro con sus incertidumbres y el ángulo de buzamiento del plano nodal de interés, sugiere que la traza superficial de la falla se encontraría entre 0,8 y 3,4 km del epicentro. Esto concuerda con la distancia obtenida de 2,5 km entre el epicentro y la traza en donde la expresión superficial posee una dirección de buzamiento similar al del plano nodal $\left(\mathrm{N} 30^{\circ} \mathrm{E}\right)$ al sur de Granadilla (FIGURA 8). La solución de este plano nodal, es además concordante con el plano de falla de dirección de buzamiento $\mathrm{N} 55^{\circ} \mathrm{E} / 25^{\circ}$ y ángulo de rake de $85^{\circ}$ observado en el afloramiento descrito en la sección anterior. Por esta razón, se considera que el origen de este sismo podría ser atribuido a la falla Cipreses.

\section{ANÁLISIS DE RESULTADOS}

\section{Interpretación}

Un esquema general del fallamiento se presenta en la FIGURA 9. En los alrededores de la falla Cipreses prevalecen tres unidades litológicas reconocidas tanto en superficie (FIGURA 3) como en la información de los pozos obtenidos del SENARA. En la parte más superficial se reconoce la Unidad Brechas (FIGURA 3 ), que incluye las brechas propiamente, sobreyacidas por una cobertura de tobas con un espesor de entre 8 y $35 \mathrm{~m}$. Las brechas corresponden con depósitos de tipo lahar y/o avalanchas de detritos, con un espesor de entre 20 a $65 \mathrm{~m}$. Debajo de esta unidad se encuentran lavas andesíticas que podrían ser parte de las formaciones Sapper o Reventado (FIGURA 3), las cuales representan las rocas inferiores observadas en los pozos del SENARA y de las cuales se desconoce su espesor. 
La interpretación propuesta es que la propagación hacia la superficie de una falla inversa desplazó y plegó estas tres unidades litológicas (FIGURA 9A) generando escarpes de flexura (e.g. Audemard, 1999, 2003; Audemard et al., 2016). Como consecuencia de la erosión de la cobertura de tobas, en los frentes de los escarpes las brechas han quedado expuestas (FIGURA
9B). Al tratarse de depósitos volcánicos caóticos es difícil observar evidencias claras del plegamiento en los afloramientos, no obstante la evidencia geomorfológica sugiere que existen al menos dos pliegues de tipo anticlinal y dos depresiones tectónicas ó pliegues de tipo sinclinal (FIGURA 9C).

A.

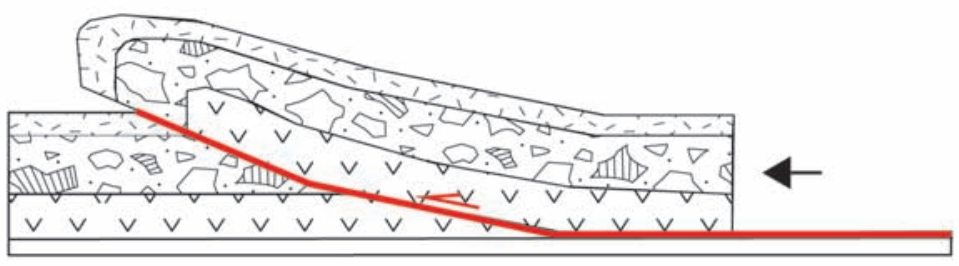

B.

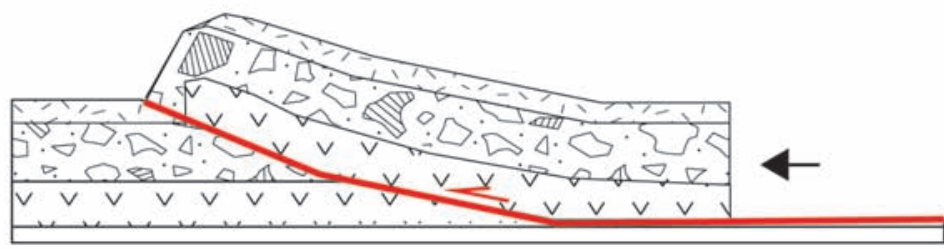

C.

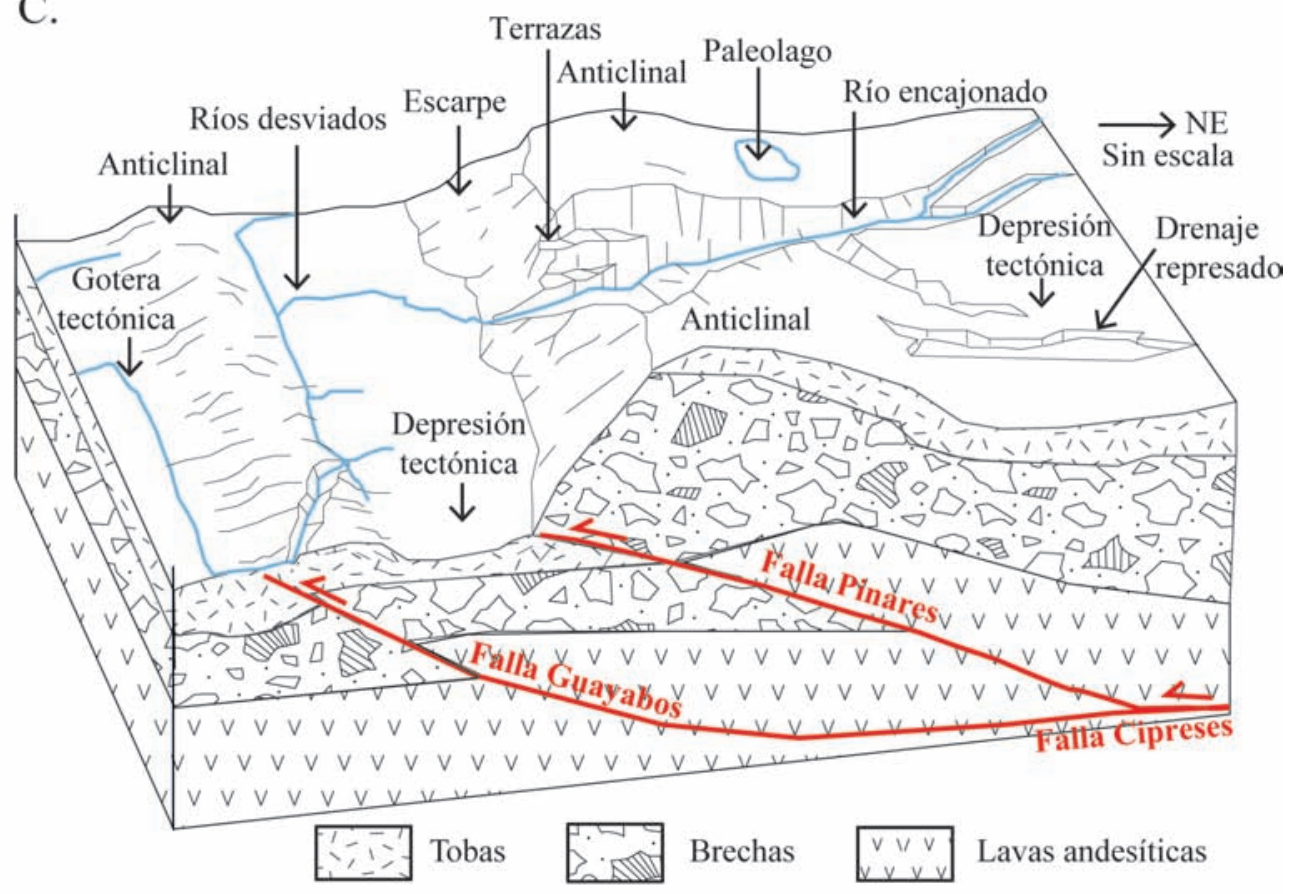

FIGURA 9. A. Modelo para el avance de un pliegue por propagación de una falla inversa. B. Modelo mostrando los efectos de la erosión al exponer las brechas del escarpe tal y como sucede en el área de estudio. C. Modelo neotectónico propuesto para la zona de estudio, mostrando los principales elementos geomorfológicos que responden al fallamiento activo. 
En el modelo propuesto, la falla Cipreses corresponde con una falla maestra que se propaga hacia la superficie a lo largo de dos ramales denominados Pinares y Guayabos, los cuales son paralelos entre sí (FIGURA 9C). La propagación de dichas fallas sería la que generó las geomorfologías descritas en la sección anterior como escarpes de flexura, contraescarpes, terrazas, gotera tectónica y ríos encajonados, desviados y represados (FIGURAS 4A, 9C). Todos estos elementos son similares a los incluidos en modelos presentados por Suppe y Medwedeff (1990), Keller y Pinter (2002), Audemard (1999, 2003) y Ollarves et al. (2006) para fallas inversas.

Se interpreta que los escarpes Pinares y Guayabos corresponden con el flanco suroeste de dos pliegues de propagación de falla de tipo anticlinal (FIGURA 8). Pliegues anticlinales similares han sido descritos relacionados con otras fallas inversas en la zona de Mérida en Venezuela (Audemard, 2003) y el Valle del Cauca en Colombia (López et al., 2009). Cada pliegue relacionado con los escarpes Pinares y Guayabos presenta además flancos traseros que corresponden con los contraescarpes que miran hacia el noreste (FIGURA 8). Esta interpretación es similar al modelo propuesto para la falla Alajuela, en el flanco suroeste del volcán Poás en la parte central de Costa Rica (FIGURA 2). En esa falla, existe un escarpe frontal y un contraescarpe de baja pendiente que representan el flanco delantero y trasero respectivamente, de un pliegue anticlinal (Borgia et al., 1990).

Las anomalías en el patrón de drenaje también se interpretan como una respuesta a la deformación cortical de la falla Cipreses. En este caso las crestas de los pliegues funcionan como obstáculos estructurales que desvían los drenajes de menor caudal pero han sido erosionados y atravesados por los ríos con mayor potencial erosivo. Las terrazas de igual manera se interpretan como el resultado del levantamiento tectónico propiciado por las fallas inversas (e.g. Audemard, 2003; Audemard et al., 2016).

De acuerdo con el análisis de la sismicidad realizado, los dos sismos del 2010 son los más cercanos a la traza de la falla Cipreses y en este estudio se considera que al menos el sismo de mayor magnitud ( $\mathrm{Mw} 4,1)$ fue originado en esta estructura ya que el mecanismo focal es de una falla inversa, con un plano nodal de dirección de buzamiento $\mathrm{N} 30^{\circ} \mathrm{E} / 35^{\circ}$ consistente con las observaciones geológicas y geomorfológicas.

\section{Potencial sísmico y zonificación}

Considerando una longitud (L) de 14,1 km, el potencial sísmico de una falla inversa de acuerdo con las ecuaciones 1 y 2 de Wells y Coppersmith (1994) mostradas en la metodología sería de una $\mathrm{Mw}$ 6,4. Usando la ecuación 3 propuesta por Stirling et al. (2008) y un ancho de deformación de 3 km (W), la magnitud sería de Mw 6,0. Finalmente, si se considera la relación propuesta por Wesnousky (2008) para fallas inversas (Ecuación 4), la magnitud sería de Mw 6,2. Otras estimaciones del potencial sísmico para la falla Cipreses fueron determinadas por Rodríguez (2017) considerando una longitud total de la falla de $8,1 \mathrm{~km}$. En ese estudio se calculó un potencial de Mw de entre 5,9 y 6,2 basado en las relaciones de Wells y Coppersmith (1994), Anderson et al. (1996) y Ambraseys y Jackson (1998).

La incertidumbre en la estimación de la longitud total de la falla $(14,1 \mathrm{~km})$ afecta directamente la determinación del potencial sísmico con el consecuente riesgo de subestimar la $\mathrm{Mw}$ en los casos en que la expresión superficial de la falla no permita determinar claramente su longitud total. Para tomar en cuenta esta situación, hemos realizado el cálculo del potencial aumentando en un $10 \%$ la longitud de la falla en cada extremo, para un total adicional de 2,8 km. Aunque es un valor arbitrario, de esta manera podemos tomar en consideración el caso de que la falla sea más larga en los extremos donde pierde expresión. El potencial sísmico usando las ecuaciones mencionadas con una longitud aumentada en 2,8 km es de Mw 6,5 (Ecuaciones 1 y 2), 6,1 (Ecuación 3) y 6,4 (Ecuación 4).

Pese a que el único sismo documentado y relacionado directamente con la falla Cipreses es de Mw baja $(4,1)$, las intensidades provocadas fueron de hasta V (IMM) generando alarma en la población de la GAM. Este sismo ejemplifica que incluso los sismos de magnitud baja originados en esta falla tienen el potencial de generar daños debido a su proximidad con la GAM y a la gran cantidad de población expuesta a la sacudida.

Costa Rica cuenta con el protocolo de zonificación alrededor de las fallas activas (Decreto 32967 del Ministerio de Ambiente y Energía) publicado en Diario Oficial La Gaceta (2006). Este protocolo promueve la restricción de construcciones ubicadas a lo largo de la traza de las fallas activas reconocidas con el fin de proteger las estructuras de una posible ruptura superficial en caso de terremoto. Pese a que en nuestro estudio se 
reconoce la ubicación de la falla Cipreses y se describe como una falla activa, la aplicación de este protocolo no es adecuada para este tipo de fallas inversas.

De acuerdo al modelo propuesto, la deformación cortical se distribuye en una zona de unos $3 \mathrm{~km}$ de ancho. Además, la expresión geomorfológica en superficie no necesariamente corresponde con la ubicación exacta de la traza de la falla, por lo que es difícil predecir en este caso la ubicación de la posible ruptura superficial durante un terremoto. Estas observaciones podrían ser usadas para sugerir un replanteamiento del protocolo de zonificación y restricción del uso del suelo en la zona inmediata a las fallas activas de tipo inverso, como la falla Cipreses.

En lugar de una restricción total alrededor de la falla, se sugiere tomar en cuenta para la zonificación el tipo y uso de las edificaciones. Ciertas estructuras vitales, como hospitales, escuelas, cárceles, etc., deberían ubicarse fuera de la zona de deformación cortical de una falla inversa. Otras edificaciones menores, sin embargo, podrían permitirse dentro de la zona de deformación. Al estar Costa Rica ubicada en una zona de alta sismicidad, lo esencial es que en cualquier parte del territorio se cumpla fielmente con el código sísmico vigente, independientemente de si las estructuras se localizan o no en la zona inmediata a una falla activa. Finalmente, en el caso de la GAM, donde se concentra la mayoría de las actividades socioeconómicas del país, es necesario el cálculo adecuado de la amenaza sísmica para favorecer el desarrollo de la misma.

\section{CONCLUSIONES}

La parte central de Costa Rica es atravesada por un sistema de fallas y plegamientos activos denominado Cinturón Deformado del Centro de Costa Rica (CDCCR), que incluye la falla Cipreses, ubicada a $5 \mathrm{~km}$ al este de San José. Los aspectos geológicos, geomorfológicos y sismológicos estudiados sugieren que esta falla es activa y de tipo inverso.

En el modelo propuesto, la falla Cipreses corresponde con una falla maestra que se propaga hacia la superficie a lo largo de dos fallas denominadas Pinares y Guayabos, las cuales son paralelas entre sí, tienen forma de "L" en vista de planta y se extienden con una longitud de $14 \mathrm{~km}$. Las principales evidencias geomorfológicas relacionadas con la falla son: escarpes de flexura, contraescarpes, terrazas, patrón de drenaje de rastrillo, goteras tectónicas y ríos encajonados, desviados y represados. Los escarpes de las fallas Pinares y Guayabos corresponden con las expresiones más prominentes de la deformación cortical y se interpretan cada uno como el flanco frontal de dos pliegues de tipo anticlinal que miran hacia el suroeste. Dos depresiones tectónicas (ó pliegues de tipo sinclinal) y contraescarpes que miran al noreste son también otros elementos geomorfológicos asociados con la deformación de estas fallas.

En términos generales, en los alrededores de la falla Cipreses prevalecen tres unidades litológicas reconocidas en superficie y en la información de los pozos del SENARA: éstas son: tobas, brechas y lavas andesíticas. La falla Cipreses desplaza y pliega estas tres unidades y como consecuencia de la erosión de las tobas sobreyacientes, las brechas se encuentran expuestas en los frentes de los escarpes Pinares y Guayabos. Una falla encontrada en un afloramiento sobrecorre las brechas sobre las tobas. Esta falla tiene una dirección de buzamiento $\mathrm{N} 55^{\circ} \mathrm{E} / 25^{\circ}$ y muestra estrías que indican un desplazamiento de tipo inverso (ángulo de rake de $85^{\circ}$ ), lo cual coincide con el modelo propuesto.

De acuerdo con el análisis de la sismicidad registrada por la RSN durante 1975-2017, los sismos en la vecindad de la falla Cipreses son escasos, aunque dos sismos ocurridos durante el 2010 tuvieron sus epicentros en las proximidades de la falla Cipreses. La solución del mecanismo focal del sismo de mayor Mw $(4,1)$ es de una falla inversa pura (ángulo de rake de $90^{\circ}$ ) con un plano nodal de dirección de buzamiento $\mathrm{N} 30^{\circ} \mathrm{E} / 35^{\circ}$. Concluimos que este sismo fue originado en la falla estudiada.

Pese a que en este estudio se reconoce la ubicación de la falla Cipreses, la deformación cortical de esta estructura se distribuye en una zona de unos $3 \mathrm{~km}$ de ancho, por lo que es difícil predecir en este caso la ubicación de la posible ruptura superficial durante un terremoto. Por esta razón se sugiere un replanteamiento del protocolo vigente de zonificación y restricción del uso del suelo en la zona inmediata a las fallas activas inversas en Costa Rica.

Aunque el único sismo documentado y relacionado directamente con la falla Cipreses es de Mw baja $(4,1)$, las intensidades provocadas fueron de hasta $\mathrm{V}$ en la escala IMM, por lo que incluso los sismos de magnitud baja tienen el potencial de generar daños menores en la GAM. Las estimaciones del potencial sísmico para la falla Cipreses indican una Mw de entre 
5,9 y 6,5. Este es un potencial sísmico peligroso para una de las fallas activas más cercanas a la GAM. El estudio detallado de esta falla y de otras ubicadas en esta zona es crucial para la apropiada determinación de la amenaza sísmica a la que se encuentra expuesta la mayoría de la población de Costa Rica.

\section{AGRADECIMIENTOS}

Este trabajo ha sido posible gracias al aporte económico que la RSN recibe proveniente de la Ley Nacional de Emergencias $\mathrm{N}^{\circ} 8488$ y a los recursos aportados por la Universidad de Costa Rica a través de los proyectos inscritos en las vicerrectorías de Investigación y Acción Social: "Vigilancia sísmica de Costa Rica" (113-B5-704), "Estudio del catálogo de sismos de la Red Sismológica Nacional” (113-B5-A02) y "Difusión de temas sismológicos de Costa Rica” (ED-3005). Agradecemos la colaboración de la Comisión Nacional de Prevención de Riesgos y Atención de Emergencias (CNE) a través de Lidier Esquivel Valverde y del Observatorio Vulcanológico y Sismológico de Costa Rica (OVSICORI-UNA) vía Floribeth Vega Solado. Agradecemos además las revisiones de José J. Martínez Díaz de la Universidad Complutense de Madrid y Franck A. Audemard Mennessier de la Fundación Venezolana de Investigaciones Sismológicas (FUNVISIS) cuyos aportes mejoraron substancialmente este artículo.

\section{REFERENCIAS}

Alonso-Henar, J., Montero, W., Martínez-Díaz, J., Álvarez-Gómez, J., Insua-Arévalo, J., and Rojas, W. (2013). The Aguacaliente Fault, source of the Cartago 1910 destructive earthquake (Costa Rica). Terra Nova, 25(5), 368-373. doi: 10.1111/ ter.12045.

Allègre, C.J., and Condomines, M. (1976). Fine chronology of volcanic processes using ${ }^{238} \mathrm{U}^{230} \mathrm{Th}$ systematics. Earth and Planetary Sciences Letters, 28(3), 395-406.

Alvarado, G.E., Vega, E., Chaves, J., y Vásquez, M. (2004). Los grandes deslizamientos (volcánicos y no volcánicos) de tipo Debris Avalanche en Costa Rica. Revista Geológica de América Central, 30, 83-99. doi: 10.15517/rgac.v0i30.7260.

Alvarado, G.E., Carr, M.J., Turrin, B.D., Swisher III, C.C., Schmincke, H.U., and Hudnut, K.W. (2006). Recent volcanic history of Irazú volcano,
Costa Rica: Alternation and mixing of two magma batches, and pervasive mixing. In: W.I. Rose, G.J.S. Bluth, M.J. Carr, J.W. Ewert, L.C. Patino, J.W. Vallance (eds.). Volcanic Hazards in Central America (pp. 259-276). The Geological Society of America Special Paper, 412.

Ambraseys, N., and Adams, R. (2001). The seismicity of Central America. A descriptive catalogue 1898-1995. Londres: Imperial College Press.

Ambraseys, N., and Jackson, A. (1998). Faulting associated with historical and recent earthquakes in the Eastern Mediterranean region. Geophysical Journal International, 133(2), 390-406. doi: 10.1046/j.1365-246X.1998.00508.x.

Anderson, J., Wesnousky, S., and Stirling, M. (1996). Earthquake size as a function of fault slip rate. Bulletin of the Seismological Society of America, 86(3), 683-690.

Audemard, F. (1999). Mopho-Structural expression of active thrust fault systems in the humid tropical foothills of Colombia and Venezuela. Zeitschrift für Geomorphologie, 118, 1-18.

Audemard, F. (2003). Geomorphic and geologic evidence of ongoing uplift and deformation in the Mérida Andes, Venezuela. Quaternary International, 101-102, 43-65. doi: 10.1016/ S1040-6182(02)00128-3.

Audemard, F., Perucca, L., Pantano, A., Ávila, C., Onorato, M., Vargas, H., Alvarado, P., and Viete, H. (2016). Holocene compression in the Acequión valley (Andes Precordillera San Juan Provinece, Argentina): Geomorphic, tectonic, and paleoseismic evidence. Journal of South American Earth Sciences, 67, 140-157. doi: 10.1016/j.jsames.2016.02.005.

Borgia, A., Burr, J., Montero, W., Morales, L.D., and Alvarado, G.E. (1990). Fault propagation folds induced by gravitational failure and slumping of the Central Costa Rica Volcanic Range: Implications for large terrestrial and Martian volcanic edifices. Journal of Geophysical Research: Solid Earth, 95(B9), 14357-14382. doi: 10.1029/JB095iB09p14357.

DeMets, C., Gordon, R.G., Argus, D.F., and Stein, S. (1994). Effect of recent revisions to the 
geomagnetic reversal time scale on estimates of current plate motions. Geophysical Research Letters, 21(20), 2191-2194. doi: 10.1029/94GL02118.

Denyer, P., y Arias, O. (1991). Estratigrafía de la región Central de Costa Rica. Revista Geológica de América Central, 12, 1-59. doi: 10.15517/rgac. v0i12.13039.

Denyer, P., Montero, W., y Alvarado, G. (2003). Atlas tectónico de Costa Rica. San José: Universidad de Costa Rica.

Diario Oficial La Gaceta. (2006). Decreto $\mathrm{N}^{\circ}$ 32967. Consultado el 3 de julio de 2018. http://www.gaceta.go.cr/pub/2006/05/04/ COMP_04_05_2006.pdf

Fernández, M., y Montero, W. (2002). Fallamiento y sismicidad del área entre Cartago y San José, Valle Central de Costa Rica. Revista Geológica de América Central, 26, 25-37. doi: 10.15517/rgac. v0i26.8528.

Fernández, M., y Pacheco, J. (1998). Sismotectónica de la región central de Costa Rica. Revista Geológica de América Central, 21, 5-23. doi: 10.15517/rgac. v0i21.8599.

Fischer, R., y Franco, J. (1979). La Formación Coris (Mioceno, Valle Central, Costa Rica). Informe Semestral Instituto Geográfico Nacional, San José, pp. 15-71.

Franco, A., Lasserre, D., Lyon-Caen, H., Kostoglodov, V., Molina, E., Guzman-Speziale, M., Monterosso, D., Robles, V., Figueroa, C., Amaya, W., Barrier, E., Chiquin, L., Moran, S., Flores, O., Romero, J., Santiago, J.A., Manea M., and Manea, V.C. (2012). Fault kinematics in northern Central America and coupling along the subduction interface of the Cocos Plate, from GPS data in Chiapas (Mexico), Guatemala and El Salvador. Geophysiscal Journal International, 189(3), 1223-1236. doi: 10.1111/j.1365-246X.2012.05390.x.

Hidalgo, P., Alvarado, G.E., y Linkimer, L. (2004). La Lavina del Valle Central (Costa Rica): ¿Lahar o Debris Avalanche?. Revista Geológica de América Central, 30, 101-109. doi: 10.15517/ rgac.v0i30.7261.
Keller, E.A., and Pinter, N. (2002). Active tectonics: earthquakes, uplift and landscape. New Jersey: Prentice Hall.

Krushensky, R.(1972). Geology of the Istarú Quadrangle, Costa Rica. Washington: USGS Bulletin.

Lienert, B.R., and Havskov, J. (1995). A computer program for locating earthquakes both locally and globally. Seismological Research Letters, 66(5), 26-36. doi: 10.1785/gssrl.66.5.26.

López, M., Moreno, M., y Audemard, F. (2009). Deformación tectónica reciente en los pie de montes de las Cordilleras Central y Occidental, valle del Cauca, Colombia. Boletín de Geología, 31(1), 11-29.

Marshall, J., Fisher, D., and Gardner, T. (2000). Central Costa Rica deformed belt: Kinematics of diffuse faulting across the western Panama block. Tectonics, 19(3), 468-492. doi: 10.1029/1999TC001136.

Montero, W. (1994). Neotectonics and related stress distribution in a subduction collisional zone: Costa Rica. In: H. Seyfried, W. Hellmann (Eds.). Geology of an Evolving Island Arc. The Isthmus of Southern Nicaragua, Costa Rica and Western Panamá (pp. 125-141). Profil 7. Stuttgart: Institut fuer Geologie und Palaeontologie der Universitaet Stuttgart.

Montero, W. (2001). Neotectónica de la región central de Costa Rica: frontera oeste de la microplaca de Panamá. Revista Geológica de América Central, 24, 29-56. doi: 10.15517/rgac.v0i24.8551.

Montero, W. (2010). El Terremoto de Cartago del 4 de mayo de 1910: Aspectos sismológicos y neotectónicos. En: G. Peraldo, B. Acevedo (eds.). Efemérides de la destrucción de Cartago cien años después (1910-2010) (pp. 37-47). San José: Ediciones Perro Azul.

Montero, W., y Alvarado, G. (1995). El terremoto de Patillos del 30 de diciembre de 1952 (Ms= $5,9)$ y el contexto neotectónico de la región del volcán Irazú, Costa Rica. Revista Geológica de América Central, 18, 25-42. doi: 10.15517/rgac. v0i18.13522. 
Montero, W., y Miyamura, S. (1981). Distribución de intensidades y estimación de los parámetros focales de los terremotos de Cartago de 1910, Costa Rica, América Central. Informe Semanal Instituto Geográfico Nacional, San José, pp. 9-34.

Montero, W., y Rojas, W. (2014). Las fallas Purires y Picagres y su relación con la secuencia sísmica de Puriscal de 1990. Revista Geológica de América Central, 50, 39-69. doi: 10.15517/RGAC. V0I50.15107.

Montero, W., Barahona, M., Rojas, W., y Taylor, M. (2005). Los sistemas de falla Agua Caliente y Río Azul y relevos compresivos asociados, Valle Central de Costa Rica. Revista Geológica de América Central, 33, 7-27. doi: 10.15517/rgac. v0i33.4233.

Montero, W., Rojas, W., y Linkimer, L. (2013). Neotectónica de las fallas Ochomogo y Capellades y su relación con el sistema de falla Aguacaliente, falda sur macizo Irazú-Turrialba, Costa Rica. Revista Geológica de América Central, 48, 119139. doi: 10.15517/rgac.v0i48.12239.

Montero, W., Linkimer, L., y Rojas, W. (2016). El sistema de Falla Navarro: desplazamientos izquierdos a lo largo del Cinturón Deformado del Centro de Costa Rica. Revista Geológica de América Central, 55, 71-100. doi: 10.15517/rgac. v55i0.27062.

Ollarves, R., Audemard, F., and Lopez, M. (2006). Morphotectonic criteria for the identification of active blind thrust faulting in alluvial environments: Case studies from Venezuela and Colombia. Zeitschrift für Geomorphologie, 145, 81-103.

Ottemöller, L., Voss, P., and Havskov, J. (2011). SEISAN: the Earthquake Analysis Software for Windows, Solaris, LINUX, and MACOSX, version 9.0.1. University of Bergen, Norway, 361p.

Peraldo, G., y Montero, W. (2010). Sismicidad anterior y posterior a los terremotos del 13 de abril y el 5 de mayo de 1910. En: G. Peraldo, y B. Acevedo (Ed.). Efemérides de la destrucción de Cartago cien años después (1910-2010) (pp. 37-47). San José: Ediciones Perro Azul.
Perucca, L., Audemard, F., Pantano, A., Vargas, H., Ávila, C., Onorato, M., Lara, G., y Esper, M. (2013). Fallas Cuaternarias con vergencias opuestas entre precordillera Central y Oriental, Provincia de San Juan. Revista de la Asociación Geológica Argentina, 70(2), 291-302.

RSN. (2010). Boletín Red Sismológica Nacional: Sismos sentidos mes de marzo 2010, San José, 2 p.

Rodríguez, J.M. (2017). Potencial sísmico de las fallas del Cinturón Deformado del Centro de Costa Rica. Tesis de Licenciatura, Escuela Centroamericana de Geología, Universidad de Costa Rica, Costa Rica.

Rojas, W. (1993). Catálogo de sismicidad histórica y reciente en América Central: Desarrollo y análisis. Tesis de Licenciatura, Escuela Centroamericana de Geología, Universidad de Costa Rica, Costa Rica.

Snoke, J., Munsey, J., Teague, A., and Bollinger, G. (1984). A program for focal mechanism determination by combined use of polarity and Sv-P amplitude ratio data. Earthquake Notes, 55, 15-20.

Stirling, M., Gerstenberger, M., Litchfield, N., McVerry, G., Smith, W., Pettinga, J., and Barnes, P. (2008). Seismic hazard of the Canterbury region, New Zealand: New earthquake source model and methodology. Bulletin of the New Zealand Society for Earthquake Engineering, 41(2), 51-67.

Stirling, M., Goded, T., Beryman, K., and Litchfield, N. (2013). Selection of earthquake scaling relationships for seismic-hazard analysis. Bulletin of the Seismological Society of America, 103(6), 2993-3011. doi: 10.1785/0120130052.

Suppe, J., and Medwedeff, D. (1990). Geometry and kinematics of fault-propagation folding. Eclogae Geologicae Helvetiae, 83(3), 409-454.

Wesnousky, S.G. (2008). Displacement and geometrical characteristics of earthquake surface ruptures: Issues and implications for seismichazard analysis and the process of earthquake rupture. Bulletin of Seismological of America, 98(4), 1609-1632. 
Wells, D.L., and Coppersmith, K.J. (1994). New empirical relationships among magnitude, rupture length, rupture width, rupture area, and surface displacement. Bulletin of Seismological of America, 84(4), 974-1002.

Woodward-Clyde Consultants. (1993). A preliminary evaluation of earthquake and volcanic hazards significant to the major population centers of the Valle Central, Costa Rica. Ret Corporation, San Francisco, 89 p.

Zárate, M.A., Mehl, A.E., and Perucca, L.P. (2014). Quaternary evolution of the Cordillera Frontal piedmont between c. 338 and 348S Mendoza, Argentina. Geological Society of London Special Publication, 399, 293-310.

\begin{tabular}{c}
\hline \hline Evelyn Rodríguez \\
ORCID: 0000-0001-9611-7980 \\
Lepolt Linkimer \\
ORCID: 0000-0002-1008-846X \\
Walter Montero \\
ORCID: 0000-0003-3092-5541 \\
\hline \hline
\end{tabular}

Trabajo recibido: julio 09 de 2018

Trabajo aceptado: marzo 06 de 2019 